\title{
Un modelo de revitalización lingüística: el caso del náhuat/ pipil de El Salvador*
}

\author{
Jorge E. Lemus \\ jlemus@udb.edu.sv \\ Universidad Don Bosco • El Salvador
}

\section{Introducción}

El náhuat o pipil es una lengua de la familia uto-azteca hablada por alrededor de 200 ancianos pipiles que habitan en algunas regiones de Sonsonate, en el occidente de El Salvador ${ }^{1}$. El náhuat ha sido clasificado como una lengua en peligro severo de extinción (ver UNESCO 1996). La última generación de náhuat-hablantes se vio forzada a dejar de transmitir su herencia lingüística a las nuevas generaciones debido a los hechos violentos de los años 1930's como resultado del levantamiento indígena y la represión y persecución gubernamental que sufrieron los pipiles en los años siguientes ${ }^{2}$. Debido a esta falta de transmisión generacional de la lengua, El Salvador perderá inevitablemente

una parte importante de su patrimonio cultural en el corto plazo si no se toman acciones concretas para su revitalización y documentación.

En este artículo se hace un resumen de la historia, cultura y lengua de este pueblo y se describen los esfuerzos de revitalización lingüística que se realizan actualmente en las zonas en las que aún hay hablantes de pipil. Se busca, a través de la enseñanza del náhuat en las escuelas, un reencuentro de las nuevas generaciones con su herencia lingüística y cultural. Se quiere a través de este programa lograr la reconstrucción de la cultura pipil y la revalorización de su contribución a la sociedad.

\footnotetext{
*Una versión anterior de este artículo fue publicada en la revista Día-logos de la Universidad Don Bosco.

${ }^{1}$ Este dato es producto de mis observaciones personales en el campo. El Censo Nacional de Población 2007 identifica únicamente a 97 hablantes.

${ }^{2}$ El 22 de enero de 1932 los indígenas y campesinos del occidente del país se levantaron con machetes y garrotes para reclamar sus tierras y mejores condiciones de vida. La bien armada y entrenada Fuerza Armada de El Salvador contrarrestó el levantamiento con lujo de barbarie. No se sabe el número exacto de rebeldes que fueron masacrados, pero algunos estimados conservadores estiman que fueron alrededor de 10,000 (Anderson 1971), mientras que otros, y la tradición oral, colocan la cifra arriba de los 30,000 (Dalton 1972). Esta masacre de indígenas y campesinos es conocida como La Matanza. Varios autores se han referido a este hecho, entre ellos sobresalen Anderson (1971) y Dalton (1972), entre otros.
} 


\section{Sobre los pipiles}

El pueblo pipil es un grupo indígena descendiente de emigrantes nahuas que llegaron a Centroamérica en diversas olas migratorias entre los años 800 y 1300 d.C., aproximadamente (ver Barberena 1966, Thompson 1948, Jiménez 1959, Fowler 1989, Torquemada 1966, Motolonía 1541, Campbell 1985, Lemus 2004). En El Salvador, estos grupos poblaron el occidente y el centro del país, en donde desarrollaron una cultura propia y se convirtieron en la cultura dominante de la región. Su idioma, el pipil o náhuat, es una lengua uto-azteca muy cercana a las demás lenguas de origen nahuatl que se hablan actualmente en México. A pesar de su gran afinidad genética, el pipil es ininteligible con estas otras lenguas náhuatl. ${ }^{3}$

La decadencia del pueblo pipil y su lengua es un proceso histórico que comenzó con la Conquista española y que los ha llevado a punto de extinción. Los pipiles actuales son un grupo étnico de difícil definición, ya que el mestizaje y el sincretismo cultural los ha vuelto un grupo que pasa desapercibido, que se confunde a simple vista con el salvadoreño promedio, y más aún con los campesinos. Las características externas encontradas en otras etnias, tales como la vestimenta y la lengua dejaron ya hace mucho tiempo de ser rasgos distintivos de los pipiles. Es por este motivo que los indígenas han sido invisibilizados por la sociedad salvadoreña en general $y$, para muchos, los grupos indígenas y sus lenguas vernáculas ya no existen en el país.

De hecho, el censo poblacional 2007 realizado por el Gobierno de El Salvador reporta que sólo el $0.2 \%$ de la población se considera indígena, lo que equivale a 11,488 personas de un total de 5,744,113 personas encuestadas. De este número, el $15.1 \%$ se considera lenca, $31.3 \%$ Cacaopera (también conocidos como kakawiras), el 26.6\% como pipiles y el resto $(27 \%)$ como pertenecientes a otras etnias. Este número ha sido cuestionado por asociaciones indígenas locales y académicos quienes consideran que el número de indígenas en este país es superior. Sin embargo, hay que tomar en cuenta que muy pocas personas se consideran a sí mismos indígenas; la mayoría perdió el vínculo cultural indígena hace ya mucho tiempo, y únicamente en algunas comunidades en el occidente y el oriente del país hay grupos pequeños que han mantenido su cultura ancestral. De estos grupos, únicamente los pipiles han mantenido la lengua.

\footnotetext{
${ }^{3}$ No solamente se ha comprobado este hecho con estudios comparativos como los llevados a cabo por Campbell (1985), sino también el autor ha propiciado reuniones con pipil-hablantes y náhuatl-hablantes en México, quienes han reportado un nivel bajo de inteligibilidad.
} 
Esta negación étnica debe hacernos reflexionar sobre quiénes son los indígenas en El Salvador. Hay dos posibilidades: i) Se es indígena por consaguinidad y ii) Se es indígena por tradición cultural. Si se es por consaguinidad, el $86.3 \%$ de la población salvadoreña que se considera mestiza, según el mismo censo citado en el párrafo anterior, podría considerarse como indígena, ya que la mayoría de salvadoreños, por el elevado mestizaje que ocurrió durante y después de la colonia, podemos considerarnos de sangre indígena. Pero si es por tradición cultural, muy pocos salvadoreños se pueden considerar culturalmente indígenas. No existe en El Salvador ni una comunidad que se pueda considerar enteramente indígena; aunque si existen comunidades, como Santo Domingo de Guzmán, Cuisnahuat, Nahuizalco e Izalco, en el departamento de Sonsonate, que tienen alta presencia indígena y que se identifican cultural y lingüísticamente como tales. Fuera de Sonsonate, son pocas las comunidades que tienen alta presencia indígena. Algunas como Tacuba (Ahuachapán) y Panchimalco (San Salvador) aún tienen vestigios culturales indígenas. Otras etnias, como los cacaoperas (Morazán) han logrado mantener algunas características culturales indígenas, aunque su lengua desapareció ya hace tiempo ${ }^{4}$. En el siguiente mapa se puede observar la distribución de los pueblos indígenas de El Salvador.

\footnotetext{
${ }^{4}$ Campbell (1975) reporta que únicamente había algunos ancianos que recordaban palabras y frases sueltas en cacaopera. "Todos son viejos y al parecer se trata de los nietos de la última generación que usaba la lengua para comunicarse" (Campbell 1975:45)
} 


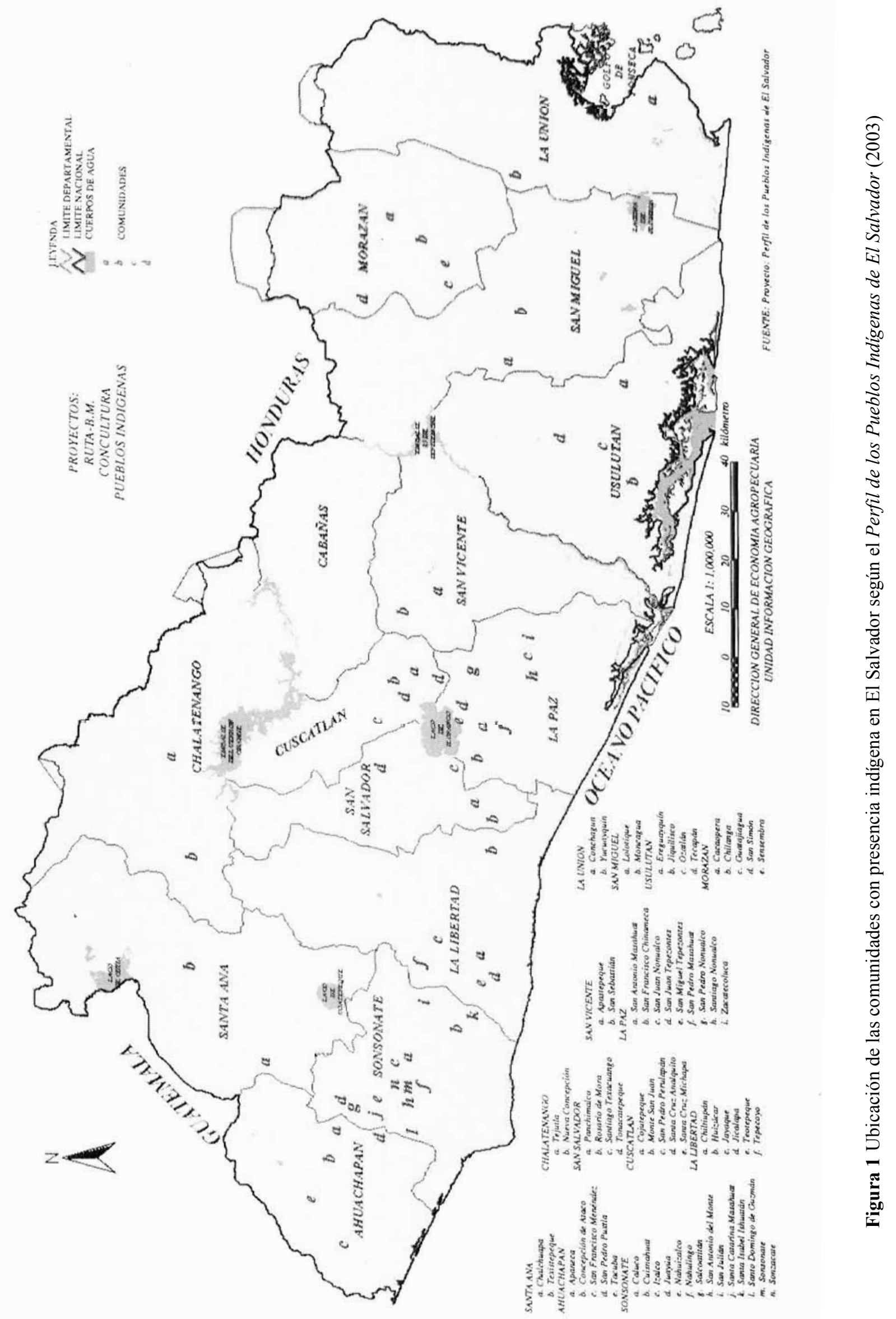


Como se muestra en la Figura 1, existen numerosas poblaciones en El Salvador en las cuales aún se pueden encontrar vestigios indígenas, aunque los habitantes estén completamente ladinizados. En general, estas comunidades ubicadas en las regiones consideradas de pobreza extrema o pobreza extrema severa según el informe de desarrollo humano en El Salvador del PNUD (2003).

\section{Situación actual de los pueblos indígenas de El Salvador}

Debido a la dificultad de identificar a los indígenas de El Salvador, el Consejo Nacional para la Cultura y el Arte (CONCULTURA) llevó a cabo una investigación que culminó con la publicación del Perfil de los Pueblos Indígenas de El Salvador (2003). Este es el único documento oficial que contiene datos estadísticos sobre los indígenas salvadoreños. En el documento se trata de tomar una fotografía del estado actual los pueblos vernáculos salvadoreños. En general, el documento muestra que los pueblos indígenas de El Salvador se encuentran en desigualdad de condiciones socioeconómicas con respecto al resto de la población del país, como se puede observar en la siguiente tabla con datos de la OPS (1999) y UNICEF (1998) $)^{5}$ publicados en el perfil.

\section{Tabla 1}

Indicadores de desarrollo

\begin{tabular}{|l|l|l|}
\hline Indicador & Promedio Nacional & Pueblos Indígenas \\
\hline vivienda con piso de tierra & $39.4 \%$ & $67.14 \%$ \\
\hline sin servicio de agua potable & $40 \%$ & $78.36 \%$ \\
\hline hogares en pobreza relativa & $25.7 \%$ & $61.1 \%$ \\
\hline hogares en pobreza extrema & $18.9 \%$ & $38.3 \%$ \\
\hline mortalidad infantil & $35 \%$ & $42.49 \%$ \\
\hline cobertura del seguro social & $17 \%$ & $3.2 \%$ \\
\hline analfabetismo & $21.5 \%$ & $35.24 \%$ (UNICEF) \\
& & $40.50 \%$ (OPS) \\
\hline
\end{tabular}

La Tabla 1 muestra el estado de pobreza y subdesarrollo en el que se encuentran los pueblos indígenas de El Salvador. Estas diferencias son el resultado de la discriminación, la explotación, la falta de oportunidades y el abuso del que han sido víctimas los indígenas salvadoreños a través de la historia. Por esta razón, los pueblos indígenas han abandonado sus costumbres y su lengua ancestrales y han adoptado la cultura y lengua mayoritarias. La vergüenza de "ser indígena" y las implicaciones sociales que eso ha tenido desde la conquista y la colonia han obligado a los indígenas salvadoreños a autoeliminarse como pueblo.

\footnotetext{
${ }^{5}$ Los indicadores estudiados por estos organismos son más de los que aquí se ejemplifican. El lector puede consultar las tablas incluidas en el Perfil de los Pueblos Indígenas (CONCULTURA et al 2003) o en los documentos de UNICEF y OPS. Para los propósitos de ejemplificar la desigualdad de condiciones entre los pueblos indígenas y el resto de la población, los datos aquí presentados son suficientes.
} 


\section{Proyecto de revitalización lingüística}

De los tres pueblos indígenas reconocidos en El Salvador, únicamente los pipiles han mantenido su lengua, aunque hablada por un grupo muy pequeño de ancianos esparcidos en pequeños poblados en el departamento de Sonsonate. Las posibilidades de revitalización de esta lengua, aunque moribunda, son reales. Las otras lenguas, lenca y cacaopera, ya no cuentan con hablantes y los datos lingüísticos de ellas son muy escasos por lo que reconstruirlas e intentar un proceso de resurrección es una tarea de casi imposible consecución. Por tal motivo, los esfuerzos de revitalización se han concentrado únicamente en el náhuat o pipil. Sin embargo, el modelo de revitalización que se describe a continuación, podría aplicarse a estos pueblos también, aunque probablemente con resultados muy limitados.

\section{Componentes del Proyecto}

La mayoría de estudios sobre las lenguas minoritarias (Fishman 1991, Wardaugh 1986, Hornberger 2003) han demostrado que cuando existen largos períodos de bilingüismo las comunidades optan por convertirse en monolingües, seleccionando el idioma de más prestigio. De hecho, esta es una decisión práctica que responde a las necesidades sociolingüísticas de la comunidad. ¿Para qué hablar dos idiomas si uno es suficiente para llevar a cabo todas las funciones sociales? ¿Por qué hablar la lengua minoritaria si ello te coloca en el nivel más bajo de la escalera social? ¿Por qué molestarse en aprender la lengua nativa si hay otras necesidades más inmediatas para la comunidad como la comida, la vivienda, la escuela, la salud, etc.? ¿Por qué aprender el idioma vernáculo si no es reconocido legalmente $\mathrm{y}$, por lo tanto, ninguna transacción comercial o legal se puede llevar a cabo en él?

La respuesta a las preguntas anteriores revela que ningún programa de revitalización lingüística puede tener éxito si no toma en cuenta otros problemas sociales de índole más práctica y no le devuelve el sentido de solidaridad, unidad e identidad al pueblo indígena. Los programas de revitalización lingüística que han tenido éxito, tales como el hebreo en Israel, el maorí en Nueva Zelanda (Spolsky 1991, 1995), el vasco en España y el friso en Holanda (Mahmood 1989) han despertado la identidad del pueblo, el sentido de solidaridad y de pertenencia. Si esta solidaridad se pierde y nunca se recupera, nunca se podrá revertir el cambio lingüístico.

A continuación se proponen cinco componentes o dimensiones que deben incluirse en el proceso de revitalización del náhuat y la revalorización de su cultura ${ }^{6}$. Los componentes son: i) identidad cultural, ii) corpus lingüístico, iii) legislación, iv) educación y v) bienestar social. No obstante la falta de desarrollo de alguno de los componentes tiene efectos negativos en el proceso de revitalización, el componente de mayor importancia es la recuperación de la identidad cultural, ya que ésta impulsa el desarrollo de los demás componentes. En este artículo, se hace mayor referencia al componente educativo y cultural del proyecto.

\footnotetext{
${ }^{6}$ La descripción de los componentes del modelo está basada enteramente en la propuesta hecha en Lemus 2004.
} 


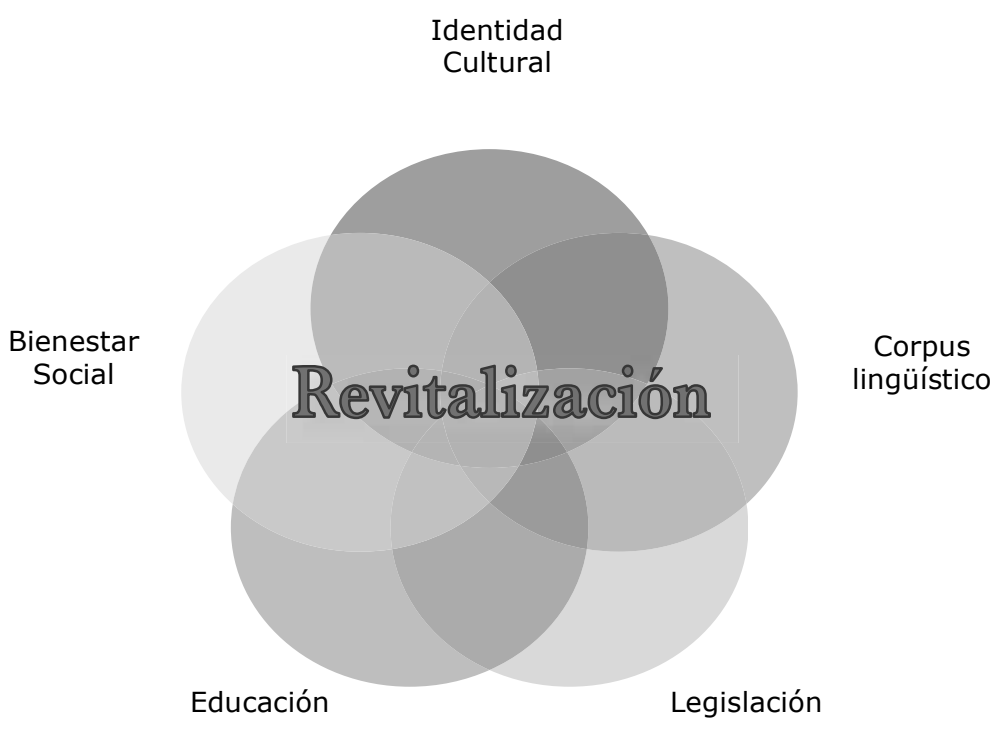

Figura 2 Componentes del proceso de revitalización lingüística (Tomado de Lemus 2004:26)

Identidad Cultural: La identidad de un pueblo está íntimamente ligada a su lengua, por lo que la pérdida de ésta pone en peligro inmediato la identidad del pueblo mismo. Varios grupos étnicos rechazan a las personas de la comunidad como indígenas cuando estos dejan de utilizar la lengua vernácula y adoptan la lengua mayoritaria. Según el poeta guatemalteco Humberto $\mathrm{Ak}^{\prime} a b a l^{7}$, en algunas comunidades indígenas guatemaltecas si los indígenas aprenden español, aunque sigan hablando la lengua materna, son considerados "ladinos" 8 por sus congéneres. Ejemplos de otros pueblos indican que la pérdida de la identidad conlleva inevitablemente a la pérdida de la lengua ${ }^{9}$. Por lo tanto, el primer paso a tomar en cualquier proceso de revitalización lingüística es reconocer la lengua como símbolo de la identidad del pueblo. El uso de la lengua, entonces, nos identifica como parte del grupo social, de la etnia en particular. Wardaugh (1986) señala que una de las primeras demandas que hacen los grupos minoritarios en cualquier parte del mundo es el derecho a usar su lengua en todos los contextos sociales. En el caso del náhuat, y debido al extenso deterioro de la lengua y a la pérdida de identidad cultural, se deben hacer grandes esfuerzos para revivir esa identidad cultural perdida y despertar en ellos el deseo de hablar náhuat y así identificarse con su etnia.

\footnotetext{
${ }^{7}$ Comunicación personal.

${ }^{8}$ Una persona "ladina" es una persona mestiza que ha abandonado sus raíces indígenas.

9 De hecho, los miles de inmigrantes salvadoreños en los Estados Unidos de América pueden dar testimonio de cómo la segunda generación (hijos de inmigrantes) pierden la lengua y la cultura, mientras que la tercera generación (los nietos), son totalmente asimilados a la cultura estadounidense. Este proceso se repite con otros grupos étnicos y en otros países.
} 
Recuperar la identidad cultural de un pueblo es una tarea extremadamente difícil cuando todos los vínculos culturales se han perdido. En El Salvador, expresiones como "se te salió el indio", "dejate de indiadas", "indio bruto", "sos bien indio", etc., son comunes entre la población para referirse a alguien cuando éste ha cometido algún error social o demuestra ignorancia. Estas expresiones son un buen ejemplo del estatus social de los indígenas por lo que nadie quiere tener nada que ver con el "indio" al que se refieren las frases anteriores. Consecuencia de esto es la negación de la identidad y la adopción total de la cultura dominante. Las capacitaciones que reciben los maestros de náhnuat en el proyecto incluyen un componente fuerte de la cultura indígena, mostrando su lado positivo y su contribución a la formación de la nación.

Bienestar social: El conocido dicho popular "Barriga llena, corazón contento" puede resumir este componente. Ya se han mencionado arriba las condiciones de extrema pobreza y de exclusión social en la que los indígenas salvadoreños viven en la actualidad, con indicadores de desarrollo humano inferiores al promedio nacional (ver Tabla 1). Habiendo sido despojados de todo, incluso su tierra ancestral, los pipiles han aprendido a valorar los bienes tangibles que les pueden proveer la satisfacción inmediata de sus necesidades. El problema de la tierra aparece primero en su agenda ya que, históricamente, siempre han dependido de ella para su subsistencia. Al quitarles los ejidos y las tierras comunales a través del Decreto Legislativo del 2 de Marzo de 1882, la economía de subsistencia del pueblo pipil colapsó y los sumió en una terrible pobreza (ver Browning 1971). El Salvador no ha ratificado la Convención 169 de la Organización Internacional del Trabajo en lo que respecta a "Pueblos Indígenas y Tribales en Países Independientes"; por lo tanto, no existe en El Salvador ninguna señal que indique que habrá una profunda reforma agraria en beneficio de los indígenas. Dada la importancia de la tierra y de otras necesidades inmediatas como la salud y la educación, el idioma no es prioridad ni representa la ambición de los pueblos indígenas. Esto indica que cualquier proceso de revitalización que se inicie, debe tomar en cuenta estos problemas. Es decir, la solución debe ser holística, y por lo tanto, debe involucrar a diversas instituciones como actores principales. Mínimamente, el programa de revitalización que aquí se propone, incluye algunos beneficios para los niños participantes, tales como golosinas, material didáctico y útiles escolares gratuitos. Los ancianos náhuat-hablantes colaboran en el aprendizaje de los maestros compartiendo historias, mitos, leyendas, anécdotas y sirviendo como consultores de la lengua. Esta colaboración es reconocida económicamente, de tal suerte que lo poco que el proyecto puede darles por su trabajo les ayude a paliar su deteriorada economía. Los recursos con los que cuenta la Universidad para este tipo de proyectos no son suficientes como para mejorar significativamente la calidad de vida de todas las comunidades indígenas, aunque se hacen esfuerzos para potenciar el desarrollo local de las mismas ${ }^{10}$.

\footnotetext{
${ }^{10}$ Este punto en particular requiere que organismos internacionales y gubernamentales se involucren en el proceso y puedan orientar y financiar el desarrollo local con una perspectiva cultural.
} 
Corpus lingüístico: Ningún esfuerzo de revitalización puede tener éxito si no se cuenta con suficientes registros y estudios lingüísticos que permitan el desarrollo de materiales para la enseñanza y estudio de la lengua. Si el idioma amenazado no cuenta con un sistema de escritura propio, se debe desarrollar uno ${ }^{11}$. También se debe normar la gramática y estandarizar la lengua, tomando uno de los dialectos como modelo. ${ }^{12}$ En el caso del pipil, ya existe un pequeño, pero significativo, corpus que ha servido de punto de partida para su desarrollo y expansión. Los más sobresalientes de estos trabajos son Jiménez (1939) quien hace un esbozo gramatical del pipil y propone una lista de toponimios, además de publicar otros trabajos etnolingüísticos sobre los pipiles; Aráuz (1960) describe el pipil de Izalco en un texto para el aprendizaje y una interpretación de de las toponimias de El Salvador; Schultze-Jena $(1977,1982)$ ofrece una colección de mitos y leyendas que él recopiló en Izalco en los años 30 y hace un intento de análisis gramatical; Rivas (1969) recupera el trabajo de Aráuz y lo sistematiza; Campbell (1985) hace una descripción de la fonología, morfología y sintaxis del pipil y además ofrece un diccionario trilingüe (españo-pipil-inglés) que es la mayor colección de vocabulario pipil existente; y Lemus (1997, 1999) muestra las discrepancias existentes entre los alfabetos utilizados para escribir pipil y propone un alfabeto simplificado, basado en el inventario fonémico del idioma, además presenta un análisis morfofonológico de la lengua y un glosario de palabras primitivas del idioma.

Todos estos análisis existentes forman la base sobre la cual se está construyendo el corpus del idioma. Se han digitalizado varios de los trabajos mencionados en el párrafo anterior y se mantiene un registro en audio y video de historias de vida, leyendas, mitos, entrevistas, diálogos, canciones, etc., de los náhuat-hablantes actuales. Este corpus sirve para el análisis lingüístico continuo del pipil y una mejor comprensión de su cosmovisión. Además, sirve de sustento teórico y provee insumos para el desarrollo de los libros de texto para el aprendizaje del náhuat y la realización de otros estudios sobre la lengua. Una vez el idioma ha sido debidamente analizado y codificado, es más fácil comenzar la producción literaria en la lengua. Hasta a fecha no existe literatura escrita originalmente en náhuat. Las historias de Schultze-Jena son transcripciones de sus entrevistas y reflejan la cosmovisión del pueblo pipil. ${ }^{13}$ Genaro Ramírez, líder y anciano náhuat-hablante pipil de Santo Domingo de Guzmán, ha publicado listas de palabras y lecciones para el aprendizaje del náhuat (ver Ramírez 1997, 2007). Se ha hecho un intento sobresaliente de darle carácter escrito al náhuat con la

\footnotetext{
${ }^{11}$ Dentro del marco del proyecto de revitalización de la lengua náhuat, se ha propuesto un nuevo alfabeto pipil el cual será utilizado en todos los trabajos sobre la lengua que se hagan con la intención de estandarizar su uso (ver ejemplo más adelante).

12 El dialecto modelo sirve únicamente de base para desarrolla la gramática y no tiene que ser necesariamente el que cuenta con el mayor número de hablantes. En algunos casos, como en Indonesia, se ha escogido un idioma y dialecto minoritario como lengua nacional, el indonesio, en lugar de las lenguas mayoritarias habladas en Yakarta, por ejemplo.

${ }^{13}$ El registro de la tradición oral es de suma importancia en el proceso de revitalización ya que nos permite registrar parte del conocimiento compartido de la comunidad y preservar ese conocimiento para nuevas generaciones.
} 
traducción al pipil de la Declaración Universal de Derechos Humanos (1997) hecha por equipo de náhuat-hablantes y lingüistas liderado por el autor de este artículo. Como se puede ver, la producción literaria en pipil es muy pobre y se centra en la enseñanza del idioma (como los trabajos de Ramírez) y no en otros géneros literarios. Uno de los retos del proyecto es la producción literaria en náhuat entre los pipiles. Inicialmente esta producción literaria será bilingüe, ya que la población náhuat-hablante es muy pequeña y los pipiles en general hablan español como su primera lengua. Lo más cercano a una producción literaria en la actualidad son las canciones que ha compuesto e interpreta Paula López, una de las náhuat-hablantes más jóvenes (alrededor de 50 años). Sus canciones están llenas de poesía y las utilizamos en el proyecto con los maestros de náhuat ${ }^{14}$. En general, se propone que cualquier tipo de literatura en náhuat que se produzca dentro y fuera del proyecto debe promover y respetar los valores culturales del pueblo pipil.

Educación: El corazón del proceso de revitalización propuesto es la educación. La recuperación de la identidad cultural y la revitalización de la lengua están centrados en las escuelas. La educación intercultural bilingüe se convierte en el bastión principal para el éxito del proceso. Esto implica un cambio en la política educativa y en los paradigmas de enseñanza de los maestros. Los casos exitosos de revitalización lingüística se han centrado en el sistema educativo. En Israel, por ejemplo, el gobierno mantiene un programa permanente para la enseñanza del hebreo a los inmigrantes llamado Ulpan; en Paraguay existe un programa de educación generalizado en guaraní; Bolivia y Perú han desarrollado sus propios programas de educación intercultural bilingüe; y en el país vasco, el uso del idioma vasco ha aumentado significativamente en la enseñanza pública y hay escuelas en las que la lengua de instrucción es el vasco. Igual sucede en Nicaragua con el Miskito y algunas lenguas mayas en Guatemala, las cuales son estudiadas en las escuelas.

En El Salvador, los náhuat-hablantes son ancianos que viven dispersos en pequeñas comunidades en el departamento de Sonsonate. La mayoría son analfabetas y viven en situación pobreza. Los náhuat-hablantes actuales nacieron en las décadas de los años 1920's y 1930's y fueron la última generación que aprendió náhuat. Éstos ya no transmitieron la lengua a las nuevas generaciones por diversos motivos sociales, como pérdida de prestigio de la lengua y la desaparición de contextos en los cuales se podía utilizar, pero especialmente por el temor a la persecución gubernamental durante los años que siguieron a la masacre de indígenas y campesinos de 1932. Los nietos y bisnietos de la última generación de náhuat-hablantes son el objetivo de este proceso de revitalización, $\mathrm{y}$, por lo tanto, receptores de los programas de revitalización que se desarrollen. Como aún viven en las comunidades, estos niños pipiles mantienen relación con los ancianos náhuat-hablantes de la comunidad, por lo que tienen la oportunidad

\footnotetext{
${ }^{14}$ Paula ha interpretado sus canciones ante diferentes públicos, incluso en la televisión nacional.
} 
de practicar en contextos sociales reales el náhuat aprendido en la escuela. De esta forma, la relación abuelo-nieto se vuelve central en el proceso de revitalización. ${ }^{15}$

Legislación: El estatus legal de un idioma determina en gran medida sus oportunidades de supervivencia. Cuando se reconoce a una lengua minoritaria como lengua nacional u oficial, el estado tiene la obligación de promover su utilización y aceptarla como una lengua legal en todos los ámbitos sociales y legales del país. No existe ninguna ley especial que proteja y promueva la utilización de las lenguas autóctonas de El Salvador; el Artículo 62 de la Constitución de la República establece que “...Las lenguas autóctonas que se hablan en el territorio nacional forman parte del patrimonio cultural y serán objeto de preservación, difusión y respeto." A pesar de este mandato constitucional, El Salvador nunca ha promovido ningún programa en beneficio de las lenguas autóctonas. Para remediar esta negligencia estatal, es necesario aprobar una "Ley de Lenguas" que verdaderamente preserve y difunda las lenguas autóctonas como lo establece la constitución. Es decir, idiomas como el náhuat deben contar con las herramientas legales que obliguen al estado a desarrollar programas que busquen revitalizar las lenguas aún habladas por minorías étnicas y documentar aquellas cuyo nivel de deterioro sea tal que revitalizarlas resulte imposible ${ }^{16}$. Las organizaciones indígenas tienen, comprensiblemente, el problema de tierras como número uno en sus agendas. Estos grupos deben incluir el problema de la lengua como algo igualmente prioritario para los pueblos indígenas. Actualmente, algunas organizaciones indígenas se han preocupado marginalmente por la lengua, por lo que es necesario generalizar esta preocupación y volverla central de los programas reivindicativos de estas organizaciones.

Todo intento por cambiar el estatus legal de la lengua debe contar con el interés de los indígenas de preservar parte importante de su identidad cultural a través del lenguaje. No es posible revitalizar una lengua cuyo pueblo no muestra interés en ella. Es por este motivo que las asociaciones indígenas ya existentes deben jugar un papel primordial en este proceso. También, instituciones educativas y culturales como las universidades, centros de investigación y ONG's deben apoyar este proceso. Esto indica que cualquier intento de cambiar el estatus legal de las lenguas autóctonas debe ser un esfuerzo conjunto de varias instituciones y asociaciones. Pero especialmente, este proceso debe contar con el interés genuino de las comunidades indígenas, quienes deben estar listas a apoyar y defender lo que es suyo. El proyecto de revitalización que se impulsa actualmente cuentan con el apoyo de las comunidades donde se desarrolla, de CONCULTURA, y se tienen conversaciones con el Ministerio de Educación para institucionalizar el programa de revitalización.

\footnotetext{
${ }^{15}$ En las escuelas más urbanas, como Izalco, son pocos los niños que tienen contacto con ancianos pipiles, pero en otras comunidades, como Santo Domingo de Guzmán y Tajcuilujlan, con mayor población indígena, existen más oportunidades.

${ }^{16}$ Por supuesto que solo la legislación a favor a de las lenguas vernáculas no es suficiente para iniciar procesos de revitalización, pero sí es un componente importante para justificar las exigencias educativas y de otra índole que puedan tener los pueblos indígenas.
} 


\section{Escuelas Participantes}

Actualmente, el programa de enseñanza del náhuat se desarrolla en 11 escuelas públicas en zonas en las que aún hay hablantes de la lengua o son reconocidas por su alta presencia indígena. Las escuelas participantes son:

Tabla 1

Escuelas Piloto

Año lectivo 2008

\begin{tabular}{|l|c|c|l|}
\hline \multicolumn{1}{|c|}{ Centro Escolar } & $\begin{array}{c}\text { Niños Estudiando } \\
\text { Náhuat }\end{array}$ & $\begin{array}{c}\text { Maestros } \\
\text { de Náhuat }\end{array}$ & \multicolumn{1}{|c|}{ Ubicación } \\
\hline 1. Dr. Mario Calvo Marroquín & 665 & 2 & Izalco, Sonsonate \\
\hline 2. Pavlo VI & 600 & 3 & Nahuizalco, Sonsonate \\
\hline 3. Anal Arriba & 115 & 1 & $\begin{array}{l}\text { Cantón Anal Arriba, } \\
\text { Nahuizalco, Sonsonate }\end{array}$ \\
\hline 4. Tajcuilujlan & 70 & 1 & $\begin{array}{l}\text { Cantón Tajcuilujlan, } \\
\text { Nahuizalco, Sonsonate }\end{array}$ \\
\hline 5. Sabana Grande & 70 & 1 & $\begin{array}{l}\text { Cantón Sabana Grande, } \\
\text { Nahuizalco, Sonsonate }\end{array}$ \\
\hline 6. Santo Domingo de Guzmán & 166 & 1 & $\begin{array}{l}\text { Santo Domingo de Guzmán, } \\
\text { Sonsonate }\end{array}$ \\
\hline 7. Tapalchukut & 89 & 1 & $\begin{array}{l}\text { Cantón Tapalchukut, Izalco, } \\
\text { Sonsonate }\end{array}$ \\
\hline 8. San Diego & 45 & 1 & $\begin{array}{l}\text { Cantón San Diego, Izalco, } \\
\text { Sonsonate }\end{array}$ \\
\hline 9. Santa Teresa & 264 & 2 & $\begin{array}{l}\text { Caserío Santa Teresa, Armenia, } \\
\text { Sonsonate }\end{array}$ \\
\hline 10. Quebrada Española & 150 & 1 & $\begin{array}{l}\text { Cantón Quebrada Española, } \\
\text { Izalco, Sonsonate }\end{array}$ \\
\hline 11. Alberto Varela & 600 & 4 & San Juan Talpa, La Paz \\
\hline TOTALES & $\mathbf{2 7 3 4}$ & $\mathbf{1 8}$ & \\
\hline
\end{tabular}

La selección de las escuelas del proyecto se basa únicamente en dos criterios: i) La escuela debe estar ubicada en una zona con alguna presencia indígena, y ii) La escuela debe mostrar interés en la revitalización y seguir los lineamientos para la enseñanza del náhuat establecidos por el proyecto; es decir, deben participar en las capacitaciones sobre náhuat y educación intercultural bilingüe ofrecidas por la universidad. Ninguna de las escuelas participantes recibe algún tipo de subsidio de parte del Ministerio de Educación (MINED) ni de otra institución pública o privada. Los maestros que reciben la capacitación para ser profesores de náhuat lo hacen voluntariamente y en su tiempo libre. Los directores y la asamblea de padres de familia también apoyan el proyecto y lo vuelven algo propio que identifica a sus escuelas de otras de la misma zona. Algunos centros escolares se han vuelto referente para otras organizaciones interesadas en la cultura salvadoreña.

Como parte del proyecto, las escuelas reciben capacitación en lengua náhuat y metodología de la enseñanza de idiomas, los libros de texto para ser utilizados en los diferentes niveles y una supervisión y asesoría continua sobre el desarrollo del programa. Para lograr esto, la Universidad Don Bosco ha establecido una oficina de la lengua 
náhuat en la Casa de la Cultura de Izalco, conocida como Tajkwiluyan ipal ne Taketzalis o TIT, que es atendida permanentemente por Carlos Cortez, un joven náhuat-hablante. Esta oficina también tiene el objetivo de brindar información sobre el proyecto o el náhuat en general a personas que lo soliciten y de dar apoyo lingüístico y metodológico a las escuelas que lo soliciten. También, en TIT tenemos el centro de documentación que puede servir de referencia para cualquier investigador.

\section{Educación Intercultural Bilingüe}

Existen diversos modelos de Educación Intercultural Bilingüe (EIB) que buscan una transición (o asimilación) menos dolorosa de la minoría étnica a la cultura dominante. En el caso de El Salvador, el sincretismo cultural que se da naturalmente al estar dos culturas en contacto, más la negación obligada de la cultura indígena entre los pipiles por la persecución de la que han sido objeto, ha invisibilizado totalmente a este grupo étnico que ha sufrido una asimilación total de su lengua y cultura a la lengua y cultura dominantes. Esto ha motivado la negación sistemática de la identidad cultural indígena y la pérdida total de la lengua al carecer ésta de contextos sociales que justifiquen su utilización.

Por tal motivo, la enseñanza de la lengua náhuat se lleva a cabo con un enfoque intercultural que motive la transformación social y la reconstrucción cultural de las comunidades en las que se desarrolla el proyecto. Se busca no solamente que los niños aprendan náhuat como lengua extranjera, sino que revaloricen su identidad indígena y desarrollen una visión crítica de la realidad que los lleve a tomar conciencia de su rol en la transformación hacia una sociedad más justa que no solamente reivindique al pueblo pipil sino también a otras minorías en situación de exclusión social. Los padres de familia y la comunidad en general son también actores importantes de este proceso ya que la transformación social involucra a todos los miembros de la comunidad.

No le podemos llamar, sin embargo, educación intercultural bilingüe en el sentido estricto al programa de enseñanza del náhuat que desarrollamos. En realidad es un acercamiento a la educación intercultural, por las características de este grupo social. No existen niños ni adultos monolingües pipiles, todos hablan español como lengua materna, y solamente un pequeño grupo de ancianos aún habla náhuat, aunque su lengua dominante es el español. El enfoque es la enseñanza de náhuat como lengua extranjera, haciendo énfasis en los valores culturales indígenas y su contribución a la sociedad, con el objeto de revalorar la cultura pipil y, a la vez, tener una mejor comprensión de las diferencias culturales existentes en su propia comunidad, no sólo con respecto a minorías lingüísticas, sino también con respecto a otras minorías, tales como las personas con discapacidades o las diferencias de género. Es decir, como todos los niños son parte de la cultura dominante, el proyecto busca desarrollar la tolerancia, la comprensión y el respeto para los grupos sociales minoritarios o excluidos por la sociedad.

\section{Libros de Texto}

Siendo el componente principal para la revitalización de la lengua el aprendizaje de ésta por niños en edad escolar, la primera tarea del proyecto fue el diseño de libros de texto para el aprendizaje del náhuat. Para ello el Departamento de Investigación de la 
universidad y un grupo de voluntarios, náhuat-hablantes, dibujantes y maestros, trabajaron en el diseño del primer set de libros para aprender náhuat ${ }^{17}$. La serie de libros se llama "Ne nawat, Tutaketzalis!” que significa "El náhuat, ¡Nuestro idioma!” y consiste de un libro de texto (llamado Amachti) y un libro de trabajo (llamado Amatzin). Además, los libros de texto están acompañados de una guía para el maestro, en la cual se desarrollan todas las unidades, con explicaciones gramaticales y sugerencias metodológicas para el desarrollo de cada una de las lecciones.

El diseño del libro está basado en un enfoque comunicativo ${ }^{18}$. El maestro desarrolla las unidades casi enteramente en lengua náhuat, obligando a los niños a interactuar en esta lengua a nivel básico. El libro introduce en las primeras lecciones las oraciones básicas a utilizar en el salón de clase por los estudiantes y el maestro. Por ejemplo, expresiones imperativas como "Siéntense", "Abran los libros", "Párense", "Escriban", "Guarden silencio", "Levanten la mano", "Escuchen", "Vean", "Salgan", etc., son aprendidas en náhuat desde el primer día por los estudiantes, de tal manera que el maestro puede interactuar en la clase sin utilizar, o utilizando en forma mínima, el español. A esto le llamamos el lenguaje básico del aula. Ni el maestro ni el alumno necesitan a este nivel hablar náhuat a la perfección para entenderse, ya que los maestros son también aprendices de náhuat. También se incluyen en el texto las expresiones más comunes necesarias para participar con éxito en ritos sociales y contextos sociolingüísticos tales como saludos, despedidas, permisos, presentaciones, preguntas permitidas, discusiones, etc.

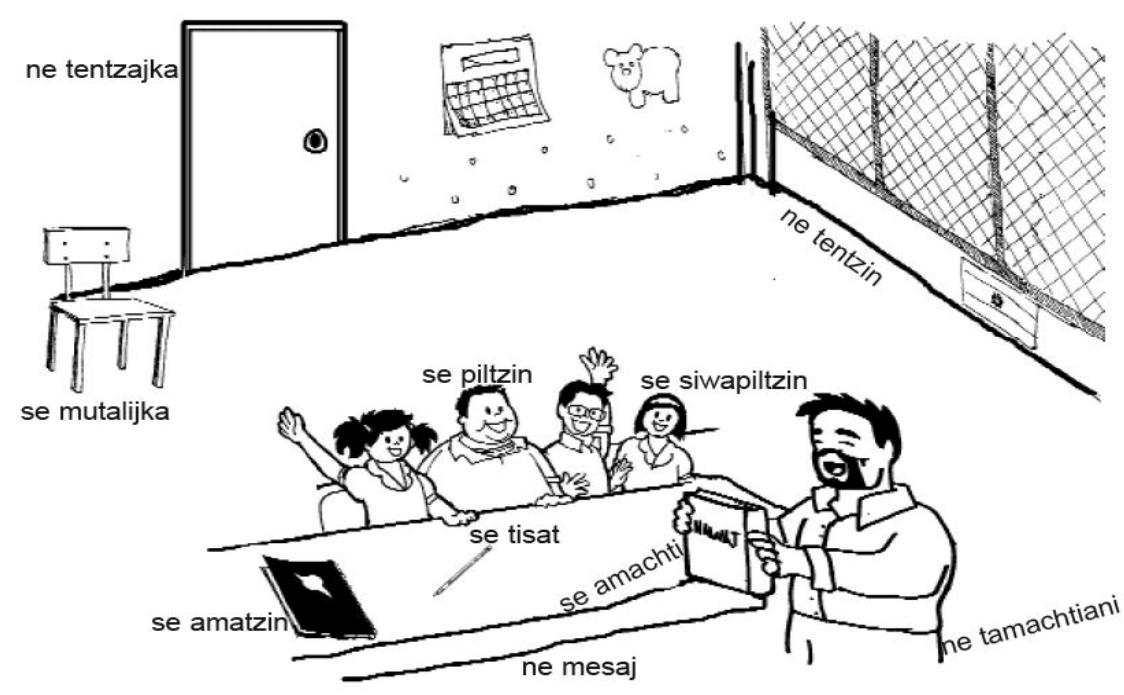

Figura 3 Lección típica para el aprendizaje del lenguaje básico del aula en los libros de texto de náhuat (Ne nawat, Tutaketzalis!).

\footnotetext{
${ }^{17}$ La coordinación del equipo para el diseño de los primeros libros estuvo a cargo de Alan King, consultor contratado por la Universidad Don Bosco para este propósito.

${ }^{18}$ Como el utilizado en los métodos para el aprendizaje de lenguas internacionales como el español y el inglés.
} 
Los textos fueron pensados originalmente para niños entre 8 y 10 años, pero la práctica ha demostrado que los libros se usan perfectamente con jóvenes y adultos. Esto ha motivado a reconsiderar el nivel de los textos y diseñar un nuevo set más apropiado para niños en las edades que cubre el proyecto hasta ahora. Las escuelas inician el programa náhuat en segundo grado y continúan hasta sexto. Se seleccionó este grado como grupo meta para iniciar el aprendizaje del náhuat ya que, en teoría, a este nivel los niños ya han internalizado el sistema de escritura del español. El proyecto no busca alfabetizar en náhuat, ya que ésta no es la lengua nativa de los niños, quienes deben ser alfabetizados en su lengua materna. El objetivo principal para trabajar con este grupo es no interferir en el proceso de aprendizaje de la escritura española y aprovechar este conocimiento para transferirlo a la escritura del náhuat, aunque el énfasis de las lecciones es la producción y comprensión oral. Además, uno de los objetivos del proyecto es influir en la generación más joven para que conozca la lengua y revalorice la cultura indígena salvadoreña. Todo esto se logra comenzando el programa educativo con los niños más pequeños. Para comenzar con los niños de primer grado tendríamos que desarrollar un texto con un enfoque completamente oral, lo cual ha sido considerado pero aún no ha sido desarrollado por falta de recursos.

\section{Estandarización lingüística}

La lengua náhuat, por el bajo status lingüístico que ha gozado desde la conquista, nunca ha contado con un alfabeto propio, sino que siempre ha sido escrita usando el alfabeto español, con los mismos valores de los grafemas (letras) españoles. Esto ha motivado que diversos estudiosos escriban el náhuat de acuerdo a su propio criterio, dando como resultado una gran variedad de formas de escribir la lengua, todas basadas en el español ${ }^{19}$. Para iniciar el proceso de revitalización, es necesario promover la estandarización de la lengua, lo cual incluye, entre otras cosas, contar con un alfabeto propio del idioma. Para ello, se adaptó el alfabeto náhuat propuesto en Lemus (1997). Debido a la cercanía fonética de la mayoría de fonemas pipiles a los fonemas españoles, el alfabeto adoptado por el proyecto tiene correspondencias directas con el español. Se puede ver en la Tabla 2 los grafemas utilizados para representar los fonemas náhuat. Consideramos que el nuevo alfabeto náhuat debería relacionarse con los valores fonéticos de los grafemas usados en español, de tal forma que se vuelva de fácil aprendizaje por los niños. En la Tabla 2 se puede observar que los únicos sonidos que son extraños al español son la palatal fricativa sorda $/ \mathrm{S} /$, la alveolar africada sorda $/ \mathrm{ts} / \mathrm{y}$ la velar labializada $/ \mathrm{k}^{\mathrm{w}} /$. El alfabeto propuesto es utilizado en todas las publicaciones en náhuat que se hacen como parte del proyecto.

\footnotetext{
${ }^{19}$ Ver Jiménez (1937), Aráuz (1960) y Todd (1953) para tener una idea de los distintos alfabetos utilizados por diferentes estudiosos. Un análisis más detallado de estos alfabetos se encuentra en Lemus (1997).
} 
Tabla 2

Alfabeto Náhuat

\begin{tabular}{|c|c|l|l}
\hline Fonema & Grafema & Ejemplo & \multirow{2}{*}{} \\
\hline$/ \mathrm{a} /$ & $\mathrm{a}$ & kal & casa \\
\hline$/ \mathrm{e} /$ & $\mathrm{e}$ & ne & el/la \\
\hline$/ \mathrm{i} /$ & $\mathrm{i}$ & tit & fuego \\
\hline$/ \mathrm{u} /$ & $\mathrm{u}$ & ume & dos \\
\hline$/ \mathrm{t} /$ & $\mathrm{ch}$ & chiwit & canasto \\
\hline$/ \mathrm{k} /$ & $\mathrm{k}$ & kumal & comal \\
\hline$/ \mathrm{l} /$ & $\mathrm{l}$ & lala & naranja \\
\hline$/ \mathrm{m} /$ & $\mathrm{m}$ & miki & morir \\
\hline$/ \mathrm{n} /$ & $\mathrm{n}$ & nan & mamá \\
\hline$/ \mathrm{p} /$ & $\mathrm{p}$ & pewa & empezar \\
\hline$/ \mathrm{k} /$ & $\mathrm{kw}$ & takwa & comer \\
\hline$/ \mathrm{s} /$ & $\mathrm{s}$ & se & uno \\
\hline$/ \mathrm{t} /$ & $\mathrm{t}$ & tutut & pájaro \\
\hline$/ \mathrm{j} /$ & $\mathrm{y}$ & yey & tres \\
\hline$/ \mathrm{w} /$ & $\mathrm{w}$ & awakat & aguacate \\
\hline$/ \mathrm{s} /$ & $\mathrm{sh}$ & padiush & gracias \\
\hline$/ \mathrm{ts} /$ & $\mathrm{tz}$ & piltzin & niño \\
\hline$/ \mathrm{h} /$ & $\mathrm{j}$ & naja & yo \\
\hline
\end{tabular}

Otro problema que se resuelve con la estandarización es la diferencia dialectal. En El Salvador existen al menos tres dialectos identificables del náhuat en las comunidades donde todavía hay hablantes. Las diferencias entre los dialectos son mínimas, pero son defendidas por sus hablantes. Una de ellas es la pronunciación de /k/, la cual en Santo Domingo de Guzmán se pronuncia como oclusiva sorda [k] o como oclusiva sonora [g], dependiendo del contexto fonético en el que se encuentre, mientras que los demás dialectos no tiene estas variantes ${ }^{20}$. Con la estandarización del idioma, las diferencias dialectales son de más fácil identificación lo que permite su estudio para dar cuenta de ellas. Además, las diferencias dialectales demuestran el dinamismo del idioma, por lo que su existencia es bien vista en términos sociolingüísticos, pero debe existir una forma estándar que permita diferenciar estos dialectos.

\section{Capacitación de Maestros}

Para alcanzar los objetivos educativos planteados anteriormente, se ha dado especial atención a la capacitación de maestros en lengua y cultura pipil, enfoques interculturales y metodología para la enseñanza de idiomas. Hasta la fecha se han capacitado más de cincuenta maestros en cinco seminarios lingüístico-metodológicos realizados en Sonsonate por la Universidad Don Bosco.

Los seminarios lingüístico-metodológicos tienen carácter intensivo y buscan darle las herramientas básicas a los nuevos maestros para que inicien la enseñanza del náhuat en

\footnotetext{
${ }^{20}$ Ver Campbell (1985) y Lemus (1997) para un estudio en mayor detalle de las diferencias dialectales.
} 
sus escuelas; además, el seminario se divide en niveles, por lo que los maestros que ya participan en el proyecto reciben capacitación continua en distintas áreas lingüísticas. Cada seminario tiene una duración de cuarenta horas y se imparte en cinco jornadas de 8 horas cada una. Los maestros que asisten a estos seminarios lo hacen en forma voluntaria y durante sus vacaciones de fin de año, debido a que durante el año lectivo no pueden abandonar sus escuelas por la escasez de maestros sustitutos y permanentes.

Los facilitadores de los seminarios son especialistas en educación bilingüe de la Universidad Don Bosco, instructores de náhuat contratados en las comunidades y profesores experimentados del proyecto. Además, se invita a ancianos náhuat-hablantes para que compartan sus experiencias con los maestros y sirvan de tutores durante el proceso de aprendizaje.

Es importante señalar que de los maestros que participan actualmente en el proyecto, solamente uno es indígena con conocimientos del náhuat, el resto son maestros que se identifican con los objetivos del proyecto y que buscan contribuir al cambio en las comunidades en las que trabajan. Este trabajo voluntario es apoyado por los directores de los centros educativos, quienes también participan en las capacitaciones y otras actividades realizadas dentro del marco del proyecto. El respaldo de los directores y los padres de familia hace posible que se impartan las clases de náhuat dentro de los horarios lectivos de los centros escolares.

\section{Profesores con poco dominio del náhuat}

Lógicamente, debido al estado moribundo del náhuat, no existen adultos jóvenes náhuathablantes que puedan fungir como maestros de náhuat en las escuelas públicas que participan en el proyecto. Los ancianos que aún hablan la lengua, no tienen las condiciones necesarias para ser maestros de aula. La mayoría son analfabetas y no se encuentran en buen estado de salud. Debido a esta realidad, el proyecto se ha enfocado en la capacitación de profesores sin conocimiento previo del náhuat para que tomen el rol de maestros náhuat. El perfil lingüístico de estos maestros es el siguiente:

- Su lengua materna es español

- Nunca antes habían estudiado náhuat/pipil

- En promedio, han recibido 40 horas de instrucción en lengua náhuat (nivel principiante)

- No hablan náhuat fluido

- Profesor-Aprendiz al mismo tiempo

Debido a la urgencia del proyecto, no podemos esperar dos o tres años hasta que los maestros alcancen un nivel de dominio del náhuat lo suficientemente alto como para sostener conversaciones fluidas en la lengua e impartir clases avanzadas enteramente en ella. Para solucionar este problema, la capacitación de los maestros se centra en el uso de un vocabulario básico para el manejo del aula, la forma de interactuar en la lengua en ritos culturales y el desarrollo de las lecciones de los libros de texto para el aprendizaje del náhuat elaborados por el proyecto. Las primeras lecciones de los libros de texto se centran en el desarrollo de vocabulario básico para el manejo del aula, de tal manera que 
tanto maestro como estudiantes puedan establecer relaciones comunicativas en náhuat sin poseer un dominio avanzado de la lengua.

Cada unidad del libro introduce un número limitado de palabras y construcciones gramaticales en náhuat que se reciclan en cada lección de la unidad. Este aprendizaje cíclico estimula la confianza del estudiante al dominar ciertas estructuras básicas que le permiten comunicarse con soltura con sus compañeros y el maestro. El uso de este vocabulario básico crea el efecto de un programa de inmersión en náhuat si se evita el uso del español en las clases. Es decir, no es necesario contar con un vocabulario extenso y un dominio avanzado del idioma para poder impartir una clase enteramente en náhuat.

También hay que señalar que el programa de náhuat comienza en segundo grado de educación básica, por lo que las clases tienden a ser más cortas y más dinámicas. La repetición constante y el uso del idioma en ambientes creados en el aula, permite que los niños internalicen muchas de las estructuras de la lengua en forma rápida. El maestro que imparte el segundo grado, es generalmente el responsable del tercer grado el siguiente año, y así sucesivamente hasta el sexto grado, por lo que su aprendizaje del náhuat coincide con el nivel de enseñanza que debe impartir. Por este motivo, los

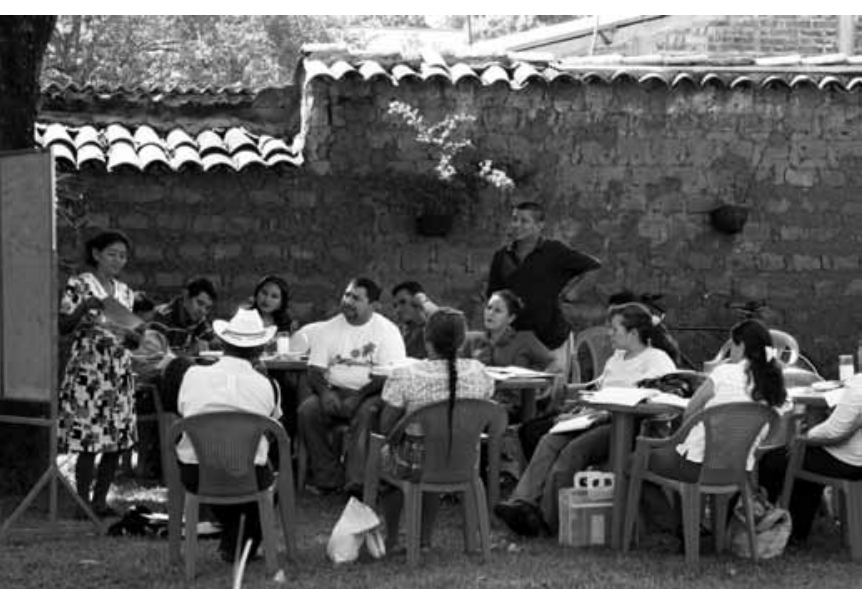

Figura 4 La náhuat-hablante pipil Paula López, de pie, enseña una canción en náhuat a los profesores del proyecto durante el V Seminario LinguisticoMetodológico realizado en Izalco, Sonsonate, en Noviembre 2007. profesores de náhuat del proyecto son también aprendices de la lengua.

El rol de los ancianos náhuat-hablantes es de consultores y tutores de la lengua. Durante las capacitaciones, al menos tres náhuat-hablantes interactúan con la clase, comparten historias, leyendas y anécdotas con los profesores y son fuente permanente de consulta sobre la lengua. La participación de los consultores náhuat en las capacitaciones, además de brindarle a los maestros modelos lingüísticos reales, permite validar los contenidos desarrollados en los libros de texto del proyecto y crea lazos de amistad con la comunidad náhuat-hablante. Se recomienda a los maestros que inviten a estos ancianos a las clases de náhuat para que los niños puedan interactuar con ellos. Esta actividad no se ha podido sistematizar aún.

\section{Las comunidades participantes}

Las comunidades que participan en el proyecto $^{21}$ han sido seleccionadas porque se considera que tienen alta presencia indígena, conservan costumbres y tradiciones pipiles

${ }^{21}$ Excepto San Juan Talpa en el departamento de La Paz, zona nonualca, en la que no hay hablantes de náhuat. 
y en ellas aún se encuentran náhuat-hablantes, lo que crea la oportunidad de interacción lingüística entre ellos y los niños en proceso de aprendizaje de esa lengua.

Esta relación abuelo-nieto, o anciano-niño, como se mencionó anteriormente, es un componente clave del programa de revitalización del náhuat. Además de los náhuathablantes que colaboran con el proyecto, se busca explotar la relación de los niños del proyecto con los ancianos de sus comunidades que todavía hablan náhuat. Generalmente, en las comunidades pequeñas, la mayoría de los niños tienen un familiar (abuelo) o conocido que habla náhuat. Esto les da la oportunidad de practicar la lengua fuera del aula, en contextos sociales reales.

La enseñanza del náhuat en San Salvador, por ejemplo, no se ha impulsado ya que la contextualización del idioma descrita arriba para las comunidades con náhuat-hablantes no se lograría. En el mediano o largo plazo se puede extender el proyecto a comunidades sin tradición indígena ni náhuat-hablantes. En el corto plazo, el proyecto está dirigido solamente a las comunidades indígenas para lograr el objetivo de revitalización.

\section{Las clases de náhuat}

Las escuelas públicas tienen un número de horas fijas para la instrucción de los estudiantes. Los programas oficiales requieren un número de horas clase para cada una de las asignaturas que se imparten en los grados. El programa de náhuat no es un programa oficial por lo que no tiene horas asignadas. Las escuelas participantes han logrado insertar en promedio una hora y media semanal de náhuat, tomando minutos de las demás asignaturas, sin afectar el desarrollo normal del currículo oficial. Esto

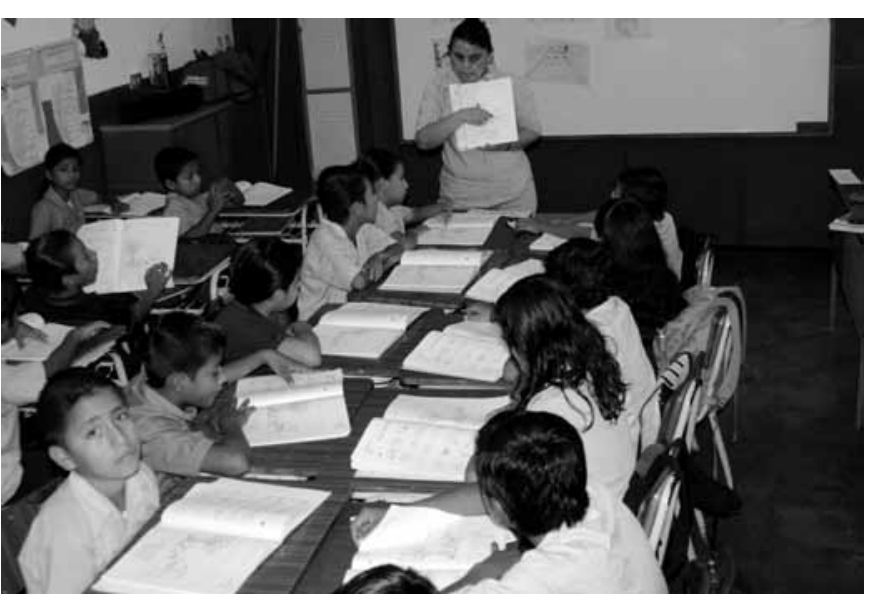

Figura 5 Niños recibiendo clases de náhuat en el CE Pavlo VI de Nahuizalco requiere trabajo extra de los maestros para cubrir los programas oficiales y además impartir clases de náhuat. Se hace énfasis, sin embargo, en la capacitaciones, el desarrollo de un currículo integrado en el cual los aspectos de la lengua y cultura pipil aparezcan como contenidos programáticos de las demás materias básicas (matemáticas, estudios sociales, lenguaje y ciencias naturales) como un eje transversal ${ }^{22}$.

Existen dos modalidades para cubrir las clases de náhuat. En la mayoría de escuelas, el profesor del grado es también el profesor de náhuat. Esta modalidad es la más común ya que la escuela no tiene que invertir en un maestro especializado, pero requiere más maestros capacitados a medida los niños avanzan a grados superiores. Además, por ser el

\footnotetext{
${ }^{22}$ No se ha logrado que ninguna escuela aplique este enfoque, aunque se está trabajando en su aplicación.
} 
mismo profesor del aula, los niños no hacen necesariamente la conexión entre idioma y maestro, porque el maestro les habla en español durante todo el tiempo en las demás clases. El cambio de un idioma a otro es más difícil en esta situación. Otras tienen uno o dos profesores de náhuat que imparten la clase en todos los grados. Estos profesores son identificados por todos los niños como tamachtiani "maestros" de náhuat, por lo que ellos relacionan al profesor con el idioma que enseña. Aunque esta modalidad es más efectiva, no todas las escuelas pueden adoptarla por los costos que implica tener un profesor dedicado. Las escuelas que tienen esta modalidad pagan el maestro con fondos propios o de la municipalidad y no con fondos del Ministerio de Educación.

El poco número de horas que se dedica a la enseñanza del náhuat (de una a dos horas semanales, máximo), hace que el proceso de revitalización camine lento y que el dominio del náhuat por los niños sea bajo. En general, los niños que comenzaron en 2004, no han alcanzado un nivel intermedio después de cuatro años de instrucción, y su dominio del idioma es todavía a nivel de principiantes. Sin embargo, se les puede escuchar hablar náhuat en los recreos y algunos reportan las "correcciones" de las que han sido objeto al intercambiar palabras con los abuelos. Esto evidencia que sí hay interacción intergeneracional en náhuat en las comunidades, aunque mínima al presente.

\section{Proyectos futuros de apoyo a la revitalización del náhuat}

El nivel de avance del proyecto en sus cinco años de ejecución ha demostrado la posibilidad real de revitalizar el náhuat. Para lograrlo, se han desarrollado otros programas dentro del proyecto. Algunos ya están en ejecución y otros aún no se han iniciado por falta de financiamiento. Algunos de estos programas se resumen a continuación.

- La Cuna Náhuat. La siguiente fase del proyecto de revitalización del náhuat es la Cuna Náhuat que consiste en la creación de un nicho lingüístico en el que niños de edad preescolar (3 a 5 años) participen en un programa de inmersión total a la lengua náhuat. Durante el tiempo de permanencia en la Cuna Náhuat, los niños participarán en diversas actividades educativas dirigidas por náhuat-hablantes de la comunidad en su lengua nativa. Es decir, mientras los niños permanecen en el centro, están totalmente expuestos a la lengua náhuat. Fuera de la cuna, se utiliza el español. Esta siguiente fase del proyecto busca crear una nueva generación de hablantes nativos del náhuat en El Salvador. La Cuna Náhuat es una especie de ludoteca comunal con un enfoque de inmersión lingüística total. Con esta modalidad se busca formar hablantes con igual grado de dominio del náhuat y el español. Se aprovechará al máximo la relación abuelo-nieto para promover el aprendizaje del náhuat en un ambiente natural.

- Nueva serie de libros. La demanda de nuevos textos para el aprendizaje del náhuat por jóvenes y adultos ha orientado el proyecto hacia el diseño de nuevos libros de texto para este grupo de aprendices. Además, se trabajará en el diseño de libros para el aprendizaje del náhuat para niños que aún no saben leer ni 
escribir. Esta serie tendrá un enfoque completamente oral y podrá ser utilizada en parvularia.

- Gramática y diccionario Náhuat. Se está trabajando en una gramática completa del náhuat que podrá ser utilizada como referencia por los maestros del proyecto y por cualquier otra persona interesada en conocer esta lengua. El proyecto también incluye un diccionario bilingüe español-náhuat, náhuat-español en versión impresa y en versión electrónica.

\section{Conclusión}

La lengua náhuat o pipil, al igual que miles de otras lenguas alrededor del mundo, se encuentra en peligro severo de extinción. Las acciones para prevenir la muerte de estas lenguas es responsabilidad de la humanidad, por lo que cualquier intento de documentación y revitalización debe contar con el apoyo de los diversos actores sociales del país. El proyecto que se ha descrito en este artículo es un intento sistemático para revertir el cambio lingüístico que ha sufrido el náhuat con respecto al español.

La enseñanza del idioma náhuat a niños escolares como parte del proyecto de revitalización de esta lengua se está llevando a cabo como una experiencia novedosa en el aprendizaje de una lengua en peligro de extinción que puede servir de modelo para lenguas minoritarias que se encuentran en similares condiciones de amenaza de extinción. Como parte del proyecto se ha desarrollado una estrategia de enseñanza del idioma náhuat a niños de segundo a sexto grado en escuelas públicas ubicadas en comunidades consideradas con presencia indígena en las cuales aún hay ancianos náhuat-hablantes. Actualmente, once escuelas públicas y más de 2500 niños reciben clases de náhuat.

El programa náhuat en las escuelas ha requerido el desarrollo de libros de texto para la enseñanza de esta lengua y la capacitación de maestros como profesores de náhuat. La Editorial Universidad Don Bosco ha iniciado la publicación de la serie de libros $\mathrm{Ne}$ Nawat, Tutaketzalis! para el aprendizaje del náhuat que incluye libro de texto, libro de trabajo y guía del maestro. Más de cincuenta profesores han sido capacitados por la universidad en educación intercultural bilingüe, lengua náhuat y metodología para la enseñanza de idiomas.

Los profesores que participan en el proyecto no tenían ningún conocimiento del náhuat al inicio del programa, por lo que el método utilizado desarrolla en ellos las habilidades lingüísticas necesarias para el manejo del aula, de tal manera que puedan impartir su clase enteramente en náhuat. Para ello, tanto maestros como alumnos aprenden una lista corta de palabras y expresiones que tienen que ver con el manejo del aula y la interacción en situaciones sociolingüísticas reales. Este enfoque es interesante ya que demuestra que el maestro no necesita ser fluido en náhuat para enseñar lo básico del idioma.

Parte esencial del proyecto es también la relación abuelo-nieto. Los abuelos son los ancianos pipiles que aún hablan la lengua pero que por su edad no pueden fungir como 
maestros, pero sí pueden ser consultores y tutores de náhuat. Los maestros jóvenes reciben constante retroalimentación sobre la lengua de los ancianos pipiles en sus comunidades y en las capacitaciones que reciben continuamente. Los niños, después de recibir clases de náhuat, tienen la oportunidad de practicar el idioma en situaciones reales con los ancianos de la comunidad. Esta relación se da principalmente en las comunidades que tienen mayor presencia indígena como Santo Domingo de Guzmán; en las demás comunidades, este relación es más limitada por el escaso número de hablantes.

En resumen, la experiencia en la enseñanza del náhuat ha demostrado que es posible iniciar un proceso de revitalización y revertir el cambio lingüístico aún cuando no hay profesores que hablen la lengua en peligro de extinción. Los maestros son profesoresaprendices de la lengua y las demandas comunicativas en el aula náhuat están acorde a su nivel de dominio de la lengua. Con la capacitación continua ofrecida por la universidad y el estudio constante de la lengua, los maestros alcanzan un nivel satisfactorio para la utilización de los textos de náhuat desarrollados por el proyecto. Además del aprendizaje del náhuat y del conocimiento de las características culturales del pueblo pipil, se está logrando la revalorización de la cultura indígena por los niños de las comunidades, con o sin ascendencia indígena, y una concientización de la sociedad sobre la problemática indígena en el país.

\section{Referencias}

Anderson, Thomas, 1971: Matanza. El Salvador's Communist Revolt of 1932, University of Nebraska Press, Nebraska.

Aráuz, Próspero (1960) El Pipil de la Región de los Itzalcos. Ministerio de Cultura, San Salvador.

Banks, James A. (2001) Cultural Diversity and Education. Foundations, Curriculum, and Teaching. Cuarta Edición. Editorial Allen and Bacon.

Browning, David (1971) El Salvador: Landscape and Society. Oxford: Clarendon Press.

Campbell, Lyle (1975) "El estado actual y la afinidad genética de la lengua indígena de cacaopera” en La Universidad, Editorial Universitaria UES, San Salvador.

(1985) The Pipil language of El Salvador. Mouton, Berlín.

(1997) American Indian Languages. The historical Linguistics of Native America. Oxford Studies in Anthropological Linguistics. Oxford University Press, Oxford

CONCULTURA et al (2003) Perfil de los Pueblos Indigenas de El Salvador. Ministerio de Educación, San Salvador.

Dalton, Roque (1972) Miguel Mármol. Los sucesos de 1932 en El Salvador. San José, Costa Rica, Editorial Educa.

Digestyc (2008) Censo de Población y Vivienda de El Salvador 2007, disponible en http://www.censos.gob.sv/

Durin, Severino (2007) “Una educación indígena intercultural para la ciudad? en Frontera Norte, vol. 19, num. 38, julio-dic

Fishman, J. A. (1991). Reversing language shift: Theoretical and empirical foundations of assistance to threatened languages. Clevedon, UK: Multilingual Matters.

García, Javier et al (1997) "La educación multicultural y el concepto de cultura" en 
Revista Iberoamericana de Educación. № 13, pp. 223-256

Hornberger, Nancy H. (2003)'The ecology of language", Gorski Lecturer, Penn State University, University Park, PA

Jiménez, Tomás Fidias (1937) Idioma Pipil de Cuzcatlán y Tunalá, hoy República de El Salvador en la América Central. Tipografía la Unión, San Salvador.

Klesing-Rempel, Úrsula (compiladora) Lo propio y lo ajeno: interculturalidad $y$ sociedad, Plaza y Valdez Editores, México

Lemus, Jorge E. (2004) "El pueblo pipil y su lengua" en Cientifica 5, Universidad Don Bosco, pp 7-28

(1997) Estudios Lingüisticos, CONCULTURA, San Salvador

Lynch, James (1986) Multicultural Education: Principles and Practice. Routledge Education Books.

Mahmood, Cynthia Keppley (1989) Frisian and Free: Study of an ethnic minority in The Netherlands. Prospect Heights, Illinois: Waveland Press, Inc.

Moya, Ruth (1997) "Interculturalidad y reforma educativa en Guatemala" en Revista Iberoamericana de Educación. $\mathrm{N}^{\mathrm{o}}$ 13, pp. 129-155

OPS, CONCULTURA, CCNIS (1999) Salud y calidad de vida de los pueblos indígenas en El Salvador. San Salvador.

PNUD (2003) Informe sobre desarrollo humano. El Salvador 2003, Desafíos y Opciones en Tiempos de Globalización, PNUD y el Informe 262: Indicadores municipales sobre desarrollo humano y los objetivos de desarrollo del Milenio

Spolsky, Bernard and R. L. Cooper (1991). The languages of Jerusalem. Oxford, Clarendon Press.

Spolsky, Bernard. (1995) Conditions for Language Revitalization: A comparison of the cases of Hebrew and Maori. Current Issues in Language and Society, 2.3.177-201

Todd, Juan G. (1953) Notas del Náhuat de Nahuizalco. San Salvador: Editorial "Nosotros".

UNESCO (1996) Atlas of the World's Languages in Danger of Disappearing, UNESCO, Paris. 